\title{
Assessment of atrial septal defect morphology by transthoracic three dimensional echocardiography using standard grey scale and Doppler myocardial imaging techniques: comparison with magnetic resonance imaging and intraoperative findings
}

Aleksandra Lange, Mohammed Walayat, Colin M Turnbull, Przemyslaw Palka, Pankaj Mankad, George R Sutherland, Michael J Godman

\begin{abstract}
Objective-To determine whether transthoracic three dimensional echocardiography is an accurate non-invasive technique for defining the morphology of atrial septal defects (ASD).
\end{abstract}

Methods-In 34 patients with secundum ASD, mean (SD) age 20 (17) years (14 male, 20 female), the measurements obtained from three dimensional echocardiography were compared to those obtained from magnetic resonance imaging (MRI) or surgery. Three dimensional images were constructed to simulate the ASD view as seen by a surgeon. Measured variables were: maximum and minimum vertical and horizontal ASD dimension, and distances to inferior and superior vena cava, coronary sinus, and tricuspid valve. In each patient two ultrasound techniques were used to acquire three dimensional data: standard grey scale imaging (GSI) and Doppler myocardial imaging (DMI). Results-Good correlation was found in maximum ASD dimension (both horizontal and vertical) between three dimensional echocardiography and both MRI (GSI $r=0.96$, SEE $=0.05 \mathrm{~cm}$; DMI $r=$ $0.97, \mathrm{SEE}=0.04 \mathrm{~cm})$ and surgery $(\mathrm{GSI} r=$ $0.92, \mathrm{SEE}=0.06 \mathrm{~cm}$; DMI $r=0.95, \mathrm{SEE}=$ $0.06 \mathrm{~cm}$ ). The systematic error was similar for both three dimensional techniques when compared to both MRI (GSI $=0.40$ cm $(27 \%) ; D M I=0.38 \mathrm{~cm}(25 \%))$ and surgery $($ GSI $=0.50 \mathrm{~cm}(29 \%) ; D M I=0.37 \mathrm{~cm}$ $(22 \%))$. A significant difference was found in both horizontal and vertical ASD dimension changes during the cardiac cycle. This change was inversely correlated with age. These findings were consistent for both DMI and GSI technique. In children (age $\leqslant 17$ years), the feasibility of detecting structures and undertaking measurements was similar for both echo techniques. However, in adult ASD patients (age $\geqslant 18$ years) this feasibility was higher for DMI than for GSI.

Conclusions-Transthoracic three dimensional imaging using both GSI and DMI accurately displayed the varying morphology, dimensions, and spatial relations of ASD. However, DMI was a more effective technique than GSI in describing ASD morphology in adults.

(Heart 1997;78:382-389)

Keywords: atrial septal defect; morphology; three dimensional echocardiography; magnetic resonance imaging

Secundum atrial septal defect (ASD) accounts for between $7 \%$ and $10 \%$ of all congenital heart disease and for between $30 \%$ and $40 \%$ of congenital heart diseases seen in adults. ${ }^{1}$ Each year approximately 300 to 400 patients in the United Kingdom undergo surgical closure of an ASD using standard sternotomy. ${ }^{2}$ The development of new techniques of ASD closure including minimal access surgery ${ }^{3} 4$ and percutaneous catheter closure ${ }^{5-8}$ has increased the need for accurate assessment not only of ASD size but also ASD morphology and its spatial relations. ${ }^{79}$ Currently, several techniques are used to image or size ASDs: echocardiography, ${ }^{11-14}$ magnetic resonance imaging (MRI), ${ }^{15}{ }^{16}$ or balloon sizing during heart catheterisation. Cross sectional echocardiography is probably the most commonly used imaging technique and with standard precordial imaging the sensitivity in detecting secundum and primum ASDs is more than $90 \% .^{14}{ }^{17}$ However, anatomically the atrial septum is a concave convex structure and therefore an ultrasound beam can cut the defect in different planes and may not reflect its true size. A potentially superior role for three dimensional echocardiography in ASD sizing was claimed recently in a comparative investigation of guided three dimensional studies and conventional cross sectional examinations. ${ }^{18}$

Previous studies on three dimensional ASD reconstruction have been carried out using standard grey scale images. ${ }^{1020}$ In these reports, because of poor transthoracic image quality grey scale images were often acquired from a transoesophageal approach. In this study, therefore, we looked not only at the potential of transthoracic three dimensional grey scale image reconstruction but at the additional value of three dimensional reconstruction using the Doppler myocardial imaging technique. Doppler myocardial imaging is based on a colour Doppler principle in which 
special algorithms are applied to detect myocardial wall motion instead of intracardiac blood flow. ${ }^{21}$ As the quality of cross sectional Doppler myocardial imaging is not directly affected by the chest wall attenuation, as it is in grey scale images, it should provide better quality images than the standard grey scale for transthoracic three dimensional echocardiography.

\section{Methods}

A prospective study of the transthoracic three dimensional echocardiographic definition of ASD morphology and its dynamic changes during the cardiac cycle was performed. Two different imaging techniques, grey scale imaging and Doppler myocardial imaging, were used to acquire three dimensional datasets from each patient. The information obtained from three dimensional Doppler myocardial imaging and grey scale images was compared with that obtained by phase contrast cine magnetic resonance imaging or surgery.

\section{PATIENT SELECTION}

Forty seven consecutive patients with a known or suspected ASD underwent cross sectional and three dimensional echocardiographic examination to determine the defect size and location. In five patients, the interatrial septum appeared to be intact on both magnetic resonance imaging and ultrasound. In seven patients no correlative measurements of the defect could be obtained because neither magnetic resonance imaging nor surgery was performed. In one patient the surgery was done elsewhere and the correlative measurements were unavailable. Therefore 34 patients, mean (SD) age 20 (17) years, with secundum ASD were suitable for study. In 18 patients, aged 23 (18) years, three dimensional grey scale images and Doppler myocardial imaging measurements were correlated with phase contrast cine magnetic resonance imaging, and in the remaining 16 patients, aged 10 (7) years, the three dimensional measurements were correlated with these taken during surgical ASD repair. The three dimensional echocardiogram and magnetic resonance imaging scans were performed on the same day in 16 patients, and in two patients there was a four day interval between the three dimensional echocardiogram and magnetic resonance imaging scan. The average time between three dimensional echocardiogram and surgery was 30 (25) days. All but two patients were in sinus rhythm. These two patients were excluded from the analysis of changes in ASD size during the cardiac cycle. All patients were informed about the purpose of the study and gave informed consent to be enrolled in the study.

THREE DIMENSIONAL ECHOCARDIOGRAPHY

The instrumentation used for the three dimensional imaging protocol consisted of an ultrasound scanner (Acuson XP10 Mountain View, California, USA) with implemented Doppler myocardial imaging software and a three dimensional acquisition system (TomTec Echo-Scan, TomTec Imaging Systems, Munich, Germany). We have described the scanner modifications which enable Doppler myocardial imaging to be acquired. ${ }^{21}$ Figure 1 shows schematically how the three dimensional

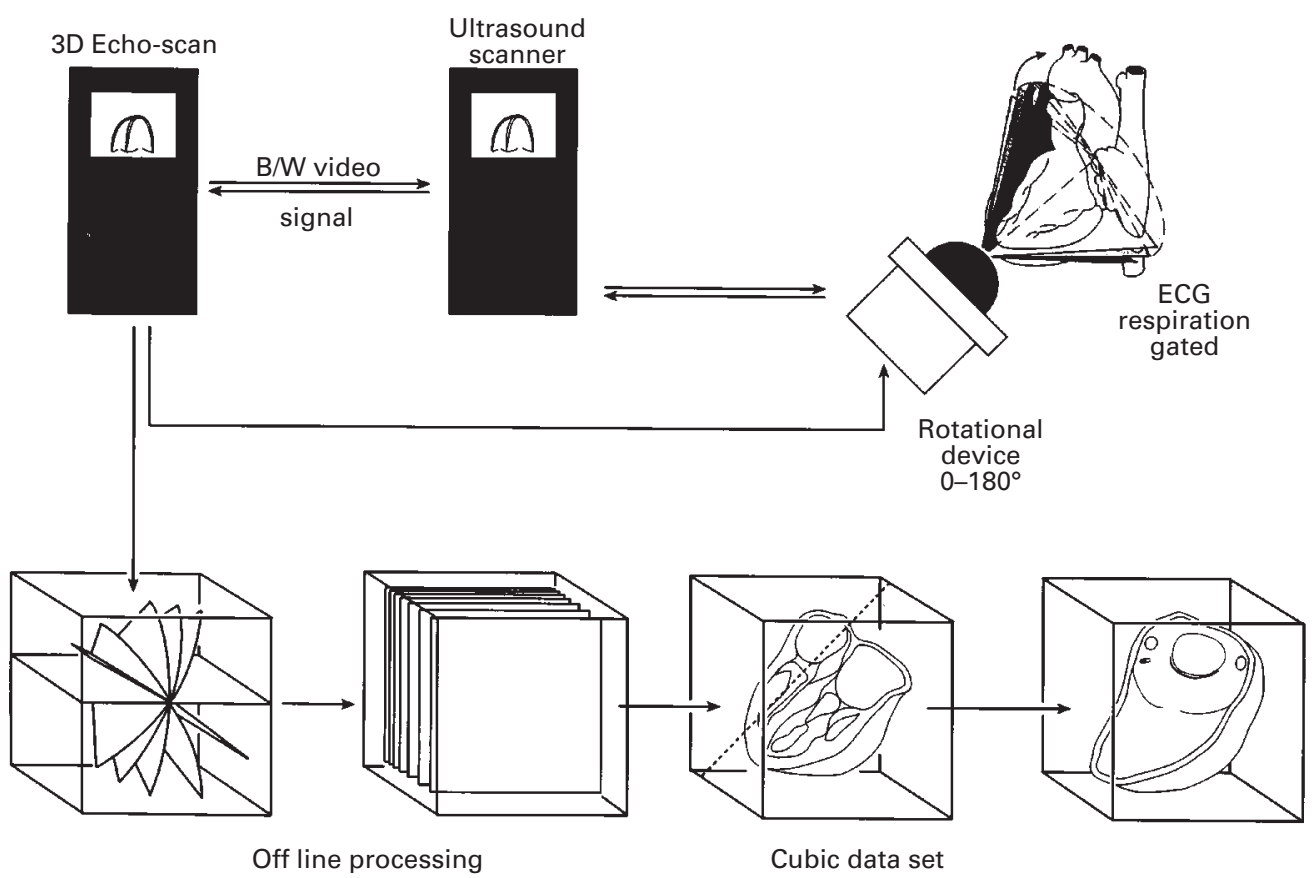

Figure 1 Steps taken to acquire three dimensional dataset. Diagram shows an ECG and respiration gated acquisition of two dimensional cross sections obtained from the apical window by rotating the transducer by the mechanical device at $2^{\circ}$ steps over $180^{\circ}$. Once the acquisition was completed, off-line processing based on the interpolation of the missing information between the acquired two dimensional images at $2^{\circ}$ steps and conversion of the images from polar to Cartesian coordinates was carried out. 


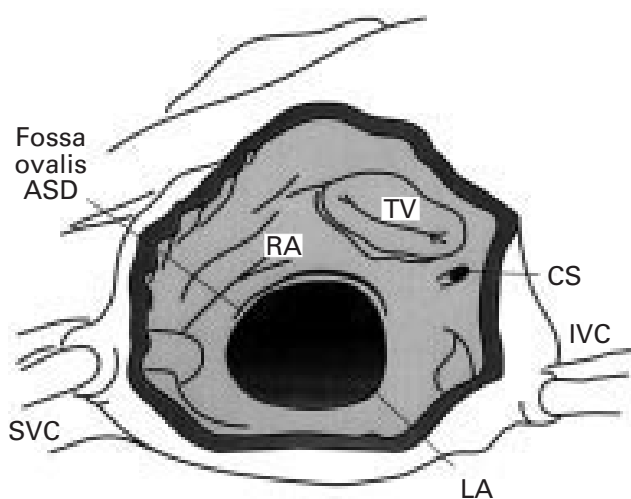

Figure 2 The schematic presentation of the atrial septal defect (ASD) as seen by the surgeon. CS, coronary sinus; $I V C$, inferior vena cava; $L A$, left atrium; $R A$, right atrium; $S V C$, superior vena cava; TV, tricuspid valve.

images were acquired. The three dimensional acquisition system consisted of a $486,66 \mathrm{MHz}$ computer with 64 megabytes of storage system memory, and steering logic for image acquisition, processing, and presentation. The ultrasound images were obtained using a 2.5-4.0 $\mathrm{MHz}$ phased array transducer steered by the transducer mechanical rotational device supplied with the Echo-Scan. In each patient the appropriate transducer frequency was selected to obtain the best quality cross sectional grey scale images. Each Doppler myocardial imaging dataset was acquired using a transducer frequency of $2.5 \mathrm{MHz}$. During the acquisition, the Echo-Scan was connected to the ultrasound video output of the Acuson scanner by a black/white video cable. Thus when Doppler myocardial images were acquired, the colour Doppler signal was transferred as a black and white video signal to the Echo-Scan. During the acquisition electrocardiographic and respiration gating was used. A standard three lead electrocardiogram cable was used to monitor the electrocardiogram while the patient's respiration was monitored by measuring skin impedance. Using this information the system created an on-line histogram based on the patient's heart rate and respiration. This enabled the setting of a gating window based on the RR intervals of the electrocardiogram. The expiratory phase was used for gating respiration. During the acquisition procedure, the transducer was placed in the standard apical position and was rotated by the mechanical rotational device at $2^{\circ}$ steps over $180^{\circ}$. Based on the gating parameters, the computer acquired one complete cardiac cycle at the acquisition start position and recorded it at 25 frames per second. When one cardiac cycle had been stored in the computer's RAM, the steering control advanced the transducer by one step. A total of 90 cardiac cycles was stored during one acquisition. The same acquisition protocol was used for the acquisition of grey scale images and Doppler images. In each patient the acquisition started with grey scale image data and then Doppler imaging data were collected. The acquisition time was approximately three minutes for both grey scale and Doppler imaging. As the patient needs to be immobile during the image acqui-
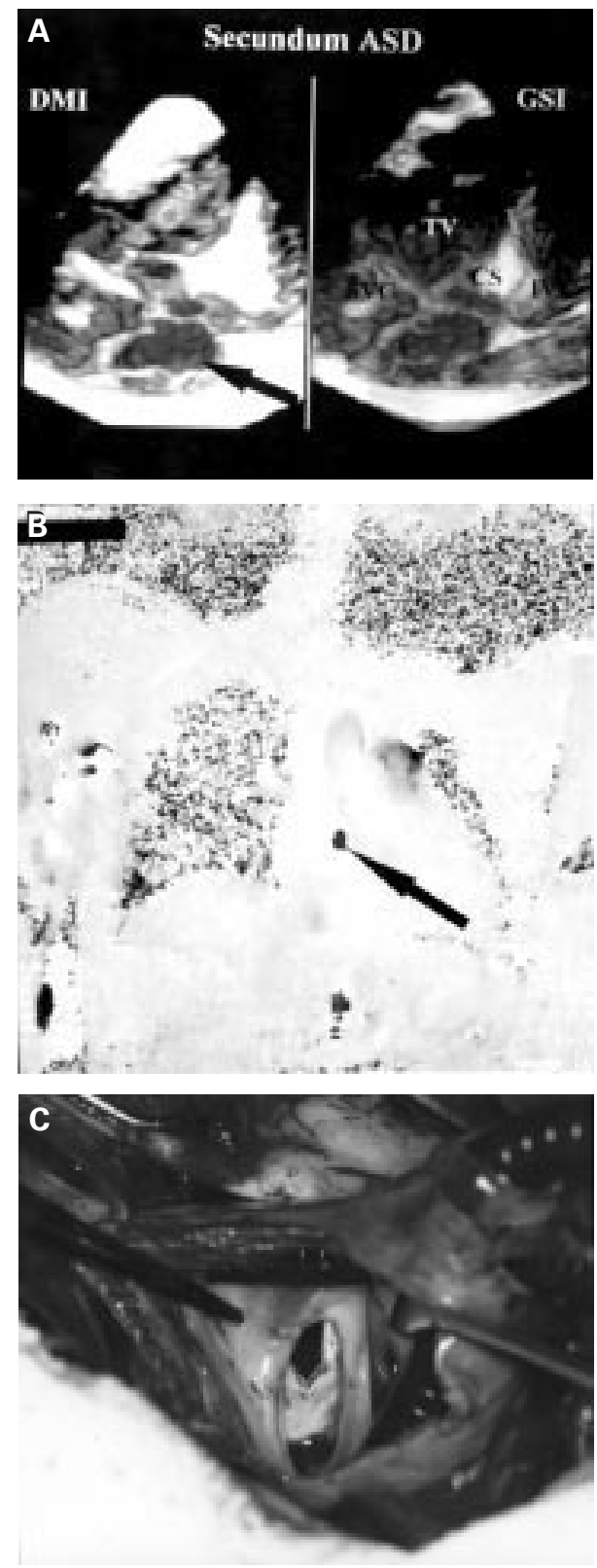

Figure 3 Maximum orifice of an atrial septal defect (ASD) as defined by three dimensional echocardiography $(A)$, phase contrast cine magnetic resonance imaging (B), and surgery $(C)$. CS, coronary sinus; DMI, Doppler myocardial imaging; GSI, grey scale imaging; IVC, inferior vena cava; SVC, superior vena cava; TV, tricuspid valve.

sition, seven patients (age between two and three and a half years) required mild sedation using Triclofos Elixir BP (triclofos sodium).

After acquisition, the data were stored on the system hard drive and then analysed off-line. In each patient, three dimensional reconstruction of the ASD was carried out from the right atrium. First, an apical four chamber view was reconstructed. Second, the acquired dataset was cut vertically by a longitudinal plane through the right atrial free wall, tricuspid valve, and anterior right ventricular free wall. Finally, the reconstruction was orientated anterior to posterior. These manoeuvres provided us with an en face view of the ASD from which the following variables were measured: minimum and maximum of horizontal and 
vertical ASD dimensions during the cardiac cycle, distances to: coronary sinus, inferior vena cava, superior vena cava, and tricuspid valve during late left ventricular systole (maximum ASD dimension) (fig 2).

Although the Doppler information is angle dependent, the angle of insonation needs to be taken into account when measuring myocardial velocities only. ${ }^{22}$ In previously reported cases where Doppler myocardial imaging was used as an acquisition technique to visualise cardiac structures, the incident angle of the ultrasonic beam did not affect the completeness of the image. This is because even very low myocardial velocities with approximate values of around 0 to $0.2 \mathrm{~m} / \mathrm{s}$ are also colour coded by Doppler myocardial imaging as a mosaic of red and blue colour. ${ }^{23} 24$

Out of the 34 defects which were reconstructed using both grey scale and Doppler images, 30 were single secundum defects and four were multiple (from two to four defects).

From all three dimensional ASD reconstructions, the feasibility of detecting right atrial structures and undertaking measurements was assessed for both Doppler myocardial imaging and grey scale imaging.

\section{MAGNETIC RESONANCE IMAGING}

The magnetic resonance imaging studies were performed on a $1.5 \mathrm{~T}$ Siemens Magneton SP system. Fast acquisition "Turboflash" localiser images (repetition time (TR) $4.9 \mathrm{~ms}$, time to echo (TE) $2 \mathrm{~ms}$, flip angle (FA) $8^{\circ}$ ) were obtained in the coronal and transverse planes through the ventricles, followed by a single angulated plane through the ventricles, and then by a single angulated plane through the interventricular and interatrial septa to give a right anterior oblique (RAO) two chamber plane localiser. Multiple contiguous, $6 \mathrm{~mm}$ slice width, 16 cardiac phases, gated cine gradient echo images (TR $560 \mathrm{~ms}$, TE $6 \mathrm{~ms}$, FA $30^{\circ}$ ) were then obtained perpendicular to

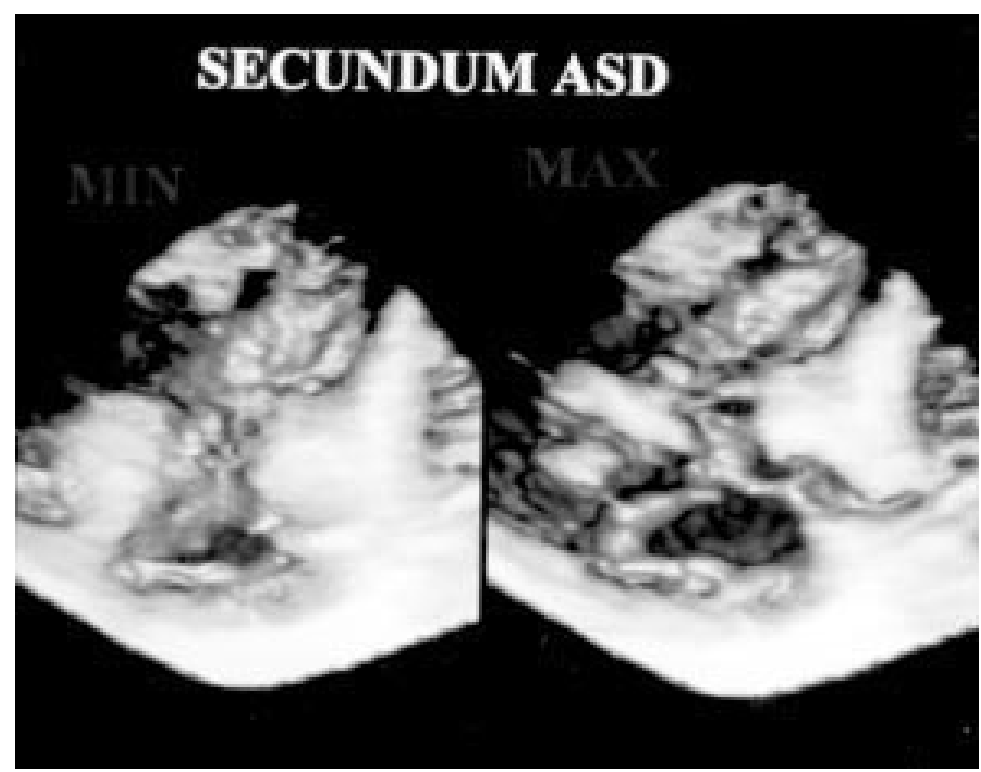

Figure 4 Changes in atrial septal defect (ASD) dimension during a cardiac cycle as seen by three dimensional Doppler myocardial imaging echocardiography. the interatrial septum in the four chamber projection, using the angulated RAO two chamber localiser.

Gated, velocity encoded, phase contrast imaging with a maximum velocity sensitivity of $120 \mathrm{~cm} / \mathrm{s}$ was then performed using four chamber cine image with the imaging plane proscribed to lie parallel to, and contiguous with, the right atrial side of the interatrial septum, to provide an en face view of the defect. The maximum dimensions of the defects were measured from the images on an independent operating console using electronic calipers.

\section{SURGICAL DATA}

All operations were performed by one surgeon. The surgery was performed either through a midline sternotomy or through a right thoracotomy, with induced ventricular fibrillation. The aorta was not cross clamped and thus the heart was not arrested in diastole using cardioplegic solution in any of the patients. Once the heart was fibrillated and the right atrium opened, a pump sucker was left in the coronary sinus and intermittent suction was performed through the ASD into the left atrium to achieve a bloodless field. The various measurements, as described above, were taken by the single observer using a string of black silk suture material which was cut at an appropriate measured point from the margin of the defect. The length of the suture was measured on a ruler and the findings were noted down by the member of the team. Two independent measurements were taken for each dimension in the first six patients. No difference was observed between the two readings and therefore in the later part of the study only one measurement was obtained in the remaining 10 patients.

\section{STATISTICAL ANALYSIS}

The data are expressed as mean (SD). A paired $t$ test was used to compare the maximum to minimum ASD dimensional change during the cardiac cycle, as measured by three dimensional echocardiography. Least square regression analysis was performed to test the correlation

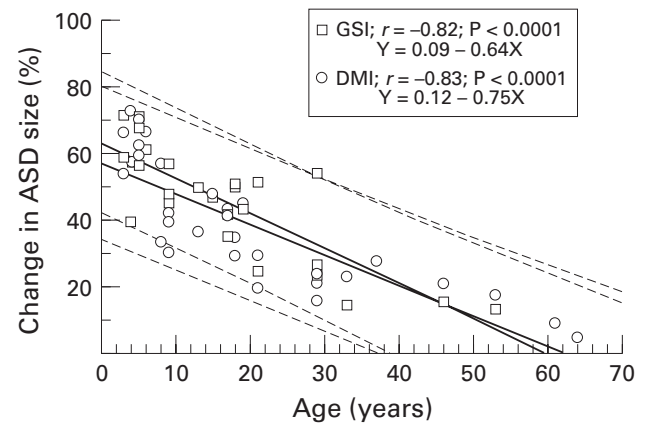

Figure 5 Linear regression analysis of the correlation between the patient's age and the dynamic changes in atrial septal defect (ASD) dimension during the cardiac cycle. The differences in ASD dimension changes were calculated as follows: [(HED - HES) + (VED - VES) / (HED + $V E D)] \times 100 \%$, where HED $=$ horizontal end diastolic dimension, HES = horizontal end systolic dimension, VED $=$ vertical end diastolic dimension, and VES = vertical end systolic dimension. Circles, Doppler myocardial imaging; squares, grey scale imaging. The dotted lines indicate the $95 \%$ predictive interval. 

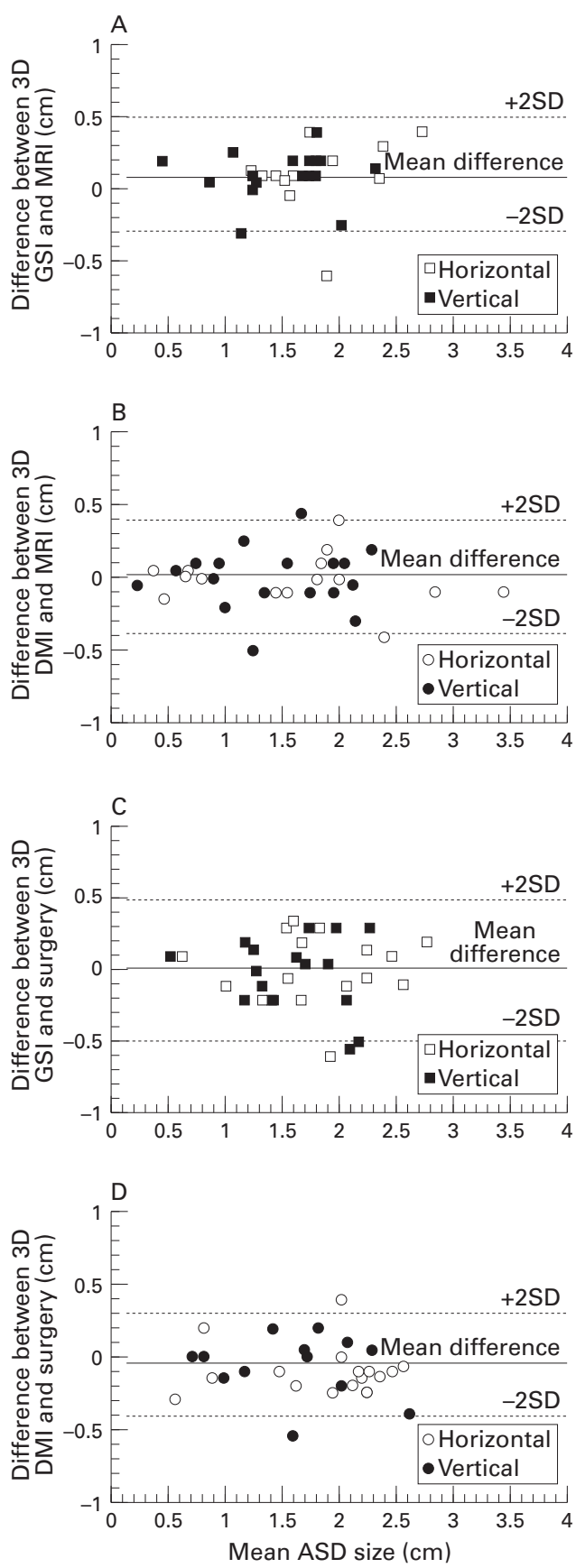

Figure 6 Bland and Altman analysis of the accuracy of atrial septal defect (ASD) dimension measurements by three dimensional echocardiography using both grey scale images and the Doppler myocardial imaging technique against phase contrast cine magnetic resonance imaging ( $A$, $B$ ) or surgery $(C, D)$. Empty squares, horizontal dimensions of $A S D$ s by grey scale images; filled squares, vertical dimensions of $A S D$ s by Doppler myocardial imaging; empty circles, horizontal dimensions of ASDs; filled circles, vertical dimensions of ASDs. The solid line shows the mean difference between the techniques used; the dotted lines show the 95\% limit of agreement.

between the horizontal and vertical dimension of an ASD, and the distances from the ASD rim to inferior vena cava, superior vena cava, coronary sinus, and tricuspid valve, measured by grey scale imaging, Doppler myocardial imaging, magnetic resonance imaging, and surgery. Linear regression analysis was performed to assess the correlation between the changes in ASD size during the cardiac cycle and age. Finally, the Bland and Altman test was used to assess: (1) the bias (systematic error) between the two ultrasound techniques and magnetic resonance imaging or surgery; (2) how the studied techniques relate to each other (underestimation or overestimation); and (3) reproducibility. ${ }^{25}{ }^{26}$ Statistical analysis was performed using the statistical package UNISTAT 4 for Windows. A $p$ value of $<0.05$ was considered significant.

\section{INTEROBSERVER AND INTRAOBSERVER} VARIABILITY

In 10 randomly selected ASD patients, all the measurements acquired from three dimensional Doppler myocardial imaging and grey scale image reconstructions pertaining to the maximum and minimum (horizontal and vertical) ASD dimensions, and distances to inferior vena cava, superior vena cava, coronary sinus, and tricuspid valve were analysed by two independent observers. Additionally, in six randomly selected ASD patients, a three dimensional echocardiographic study was performed twice within an average period of 28 (4) days. Analysis of variance was used to assess the differences between the measurements of ASD morphology obtained by two observers (interobserver variability) and between measurements taken from the same subjects at different times (intraobserver variability). Both intraobserver and interobserver variability in ASD morphology are presented as the mean (SD).

The interobserver variability for three dimensional echocardiography for grey scale images was at $0.08(0.09) \mathrm{cm}$ (systematic error $16 \%)$ and for Doppler myocardial imaging, $0.07(0.08) \mathrm{cm}$ (systematic error 13\%).

The intraobserver variability for grey scale images was at $0.07(0.08) \mathrm{cm}$ (systematic error $15 \%)$ and for Doppler myocardial imaging $0.06(0.08) \mathrm{cm}$ (systematic error $12 \%$ ).

\section{Results}

Figure 3 shows the maximum ASD orifice as defined by three dimensional echocardiography using both techniques (Doppler myocardial imaging and grey scale images), magnetic resonance phase contrast cine imaging, and surgery.

Table 1 presents the measurements of ASD dimensions and the distances from the ASD rim to other structures of the right atrium by three dimensional echocardiography, magnetic resonance imaging, and surgery. A significant difference was found in changes of both horizontal and vertical ASD dimensions during the cardiac cycle (table 1, fig 4). The maximum dimension of ASDs was found in late ventricular systole and the minimum in late ventricular diastole. Stepwise multivariate regression analysis showed that the changes in ASD size are not dependent on the defect size but are inversely related to patient age. Figure 5 presents the linear regression analysis of the relation between the changes in ASD size and patient age.

A good correlation was obtained between maximum horizontal and vertical ASD dimensions by three dimensional echocardiography 
Table 1 The measurements of atrial septal defect (ASD) dimensions and the distances from the ASD rim to other structures of the right atrium

\begin{tabular}{|c|c|c|c|c|c|c|c|c|}
\hline \multirow[b]{3}{*}{ Mean $(S D)(\mathrm{cm})$} & \multicolumn{4}{|l|}{ Dimension } & & & & \\
\hline & \multicolumn{2}{|l|}{ Maximum } & \multicolumn{2}{|l|}{ Minimum } & \multicolumn{4}{|c|}{ Distance to } \\
\hline & $H D$ & $V D$ & $H D$ & $V D$ & $I V C$ & $S V C$ & $C S$ & $T V$ \\
\hline GSI & $1.9(0.8)^{\star}$ & $1.6(0.6)^{\star}$ & $1.2(0.7)$ & $0.9(0.5)$ & $1.4(0.5)$ & $1.1(0.5)$ & $0.9(0.5)$ & $2.1(0.6)$ \\
\hline DMI & $1.8(0.7)^{\star}$ & $1.6(0.6)^{\star}$ & $1.2(0.7)$ & $0.9(0.6)$ & $1.5(0.8)$ & $1.0(0.4)$ & $1.0(0.5)$ & $2.3(0.7)$ \\
\hline MRI & $1.7(0.8)$ & $1.4(0.6)$ & N/A & N/A & N/A & N/A & N/A & N/A \\
\hline Surgery & $1.9(0.6)$ & $1.7(0.5)$ & N/A & N/A & $1.3(0.6)$ & $0.8(0.3)$ & $0.9(0.4)$ & $1.9(0.7)$ \\
\hline Mean & $1.8(0.8)^{\star}$ & $1.6(0.6)^{\star}$ & $1.2(0.7)$ & $0.9(0.5)$ & $1.4(0.7)$ & $1.0(0.4)$ & $0.9(0.5)$ & $2.2(0.6)$ \\
\hline
\end{tabular}

CS, coronary sinus; DMI, Doppler myocardial imaging; GSI, grey scale imaging; HD, horizontal dimension; IVS, inferior vena cava; MRI, magnetic resonance imaging; SVC, superior vena cava; TV, tricuspid valve; VD, vertical dimension. ${ }^{\star} \mathrm{p}<0.001 v$ minimum.

Table 2 Correlation between the measurements obtained by three dimensional echocardiography and surgery

\begin{tabular}{|c|c|c|c|c|c|c|c|c|}
\hline & \multicolumn{4}{|l|}{ GSI } & \multicolumn{4}{|l|}{$D M I$} \\
\hline & $r$ & Coefficient & $\begin{array}{l}\text { Standard } \\
\text { error }(\mathrm{cm})\end{array}$ & $p$ value & $r$ & Coefficient & $\begin{array}{l}\text { Standard } \\
\text { error }(\mathrm{cm})\end{array}$ & $p$ value \\
\hline IVC & 0.84 & 0.82 & 0.19 & 0.0025 & 0.92 & 0.96 & 0.12 & 0.0001 \\
\hline SVC & 0.64 & 0.57 & 0.21 & 0.0198 & 0.73 & 1.03 & 0.27 & 0.0023 \\
\hline CS & 0.68 & 1.39 & 0.50 & 0.0223 & 0.96 & 0.98 & 0.08 & 0.0001 \\
\hline TV & 0.82 & 0.75 & 0.15 & 0.0004 & 0.86 & 0.94 & 0.15 & 0.0001 \\
\hline Mean (SD) & $0.75(0.08)$ & $0.88(0.31)$ & $0.26(0.14)$ & $0.0113(0.001)$ & $0.87(0.09)$ & $0.98(0.03)$ & $0.16(0.07)$ & $0.0007(0.001)$ \\
\hline
\end{tabular}

CS, coronary sinus; DMI, Doppler myocardial imaging; GSI, grey scale imaging; IVC, inferior vena cava; SVC, superior vena cava; TV, tricuspid valve.

and magnetic resonance imaging (grey scale images: $r=0.96 \mathrm{~cm}$, SEE $=0.05, \mathrm{p}<0.001$; Doppler myocardial imaging: $r=0.97 \mathrm{~cm}, \mathrm{SEE}$ $=0.04, \mathrm{p}<0.001$ ) or surgery (grey scale images: $r=0.92 \mathrm{~cm}$, SEE $=0.06, \mathrm{p}<0.001$; Doppler myocardial imaging: $r=0.95, \mathrm{SEE}=$ $0.06, \mathrm{p}<0.001)$. Figure 6 shows the difference in ASD size as determined by three dimensional echocardiography and magnetic resonance imaging or surgery using the Bland and Altman analysis. The systematic error (bias) between three dimensional echocardiography and magnetic resonance imaging was low at $0.40 \mathrm{~cm}(27 \%)$ for grey scale images and 0.38 $\mathrm{cm}(25 \%)$ for Doppler myocardial imaging. For surgery the systematic error was at $0.50 \mathrm{~cm}$ $(29 \%)$ and $0.37 \mathrm{~cm}(22 \%)$ respectively. Good correlation was also obtained between the distances from the defect rim to inferior vena cava, superior vena cava, coronary sinus, and tricuspid valve in its maximum opening by three dimensional echocardiography and surgery (table 2).

In children (from three to 17 years of age; 19 patients) the feasibility of detecting structures and undertaking measurements was similar for

Table 3 Feasibility of detecting structures and undertaking measurements from three dimensional atrial septal defect (ASD) reconstructions by grey scale imaging (GSI) and Doppler myocardial imaging (DMI)

\begin{tabular}{|c|c|c|c|c|c|c|c|c|}
\hline & \multicolumn{4}{|c|}{$\begin{array}{l}\text { Group 1, mean age } 8 \text { (5) years } \\
(n=19)\end{array}$} & \multicolumn{4}{|c|}{$\begin{array}{l}\text { Group } 2, \text { mean age } 35(15) \text { years } \\
(n=15)\end{array}$} \\
\hline & \multicolumn{2}{|l|}{$G S I$} & \multicolumn{2}{|l|}{$D M I$} & \multicolumn{2}{|l|}{ GSI } & \multicolumn{2}{|l|}{$D M I$} \\
\hline & $n$ & $\%$ & $n$ & $\%$ & $n$ & $\%$ & $n$ & $\%$ \\
\hline \multicolumn{9}{|l|}{ Maximum } \\
\hline \multicolumn{9}{|l|}{ Minimum } \\
\hline dimension & 16 & 84 & 18 & 95 & 11 & 73 & 14 & 93 \\
\hline SVC & 13 & 68 & 15 & 79 & 10 & 67 & 12 & 80 \\
\hline IVC & 14 & 74 & 16 & 84 & 11 & 73 & 12 & 80 \\
\hline $\mathrm{CS}$ & 12 & 63 & 16 & 84 & 8 & 53 & 12 & 80 \\
\hline $\mathrm{TV}$ & 19 & 100 & 19 & 100 & 12 & 80 & 15 & 100 \\
\hline Mean (SD) & $15(3)$ & $82(15)$ & $17(2)$ & $90(8)$ & $11(1)$ & $71(9)$ & $13(1)$ & $89(9)$ \\
\hline
\end{tabular}

CS, coronary sinus; IVC, inferior vena cava; SVC, superior vena cava; TV, tricuspid valve. both Doppler myocardial imaging and grey scale imaging. In adult ASD patients (from 18 to 61 years of age; 15 patients), the feasibility was better for Doppler myocardial imaging than for grey scale imaging (table 3 ).

\section{Discussion}

With the growing interest in new techniques of ASD closure, precise assessment of ASD is crucial for optimal patient selection. Cross sectional echocardiography and magnetic resonance imaging are the two most commonly used techniques to assess ASD size and morphology. The accuracy of magnetic resonance imaging is well established. ${ }^{27}{ }^{28}$ It is claimed to have a sensitivity and specificity greater than $90 \%$ in the identification of ostium secundum ASD and to be superior to standard transthoracic and transoesophageal cross sectional echocardiography for ASD sizing. ${ }^{16}$

During the last 15 years dynamic research has been conducted in the development of three dimensional echocardiography which may become a bedside diagnostic technique in the assessment not only of ASD size and morphology but also of spatial relations of the defect to other cardiac structures. ${ }^{29-31}$ This seems to be particularly important in selecting patients for percutaneous ASD closure by transcatheter device placement. ${ }^{12}$ Preliminary studies have been carried out by others showing the ability of three dimensional echocardiography to reconstruct en face the dynamic morphology of ASDs using transthoracic or transoesophageally acquired ultrasound data. ${ }^{19} 2032$ However, no comparison has been made to define the accuracy of these reconstructions. It was reported that the quality of transthoracic standard ultrasound images was not satisfactory in all cases and the transthoracic three dimensional en face reconstruction of ASD was feasible in $81 \%$ of a study group of children. ${ }^{20}$ 
In our study we therefore looked not only at the potential accuracy of transthoracic three dimensional grey scale image reconstruction but also at the additional value of three dimensional reconstruction using the Doppler myocardial imaging technique. We have shown that the feasibility of detecting right atrial structures and undertaking measurements by the two techniques studied was different with age. In children ( $\leqslant 17$ years of age) all the required anatomical structures were reconstructed in a similar percentage of patients. However, in patients over 18 years old, the feasibility of detecting anatomical structures was greater with Doppler imaging. For both age groups, superior vena cava and coronary sinus were the hardest to reconstruct by either ultrasound technique.

We have also shown that all the ASDs studied changed significantly in dimension during the cardiac cycle, with maximum size in late left ventricular systole and minimum in late left ventricular diastole. Although, this difference was present in all patients, it was inversely correlated with age. A similar finding of a significant difference in ASD area during the cardiac cycle has been reported by others. ${ }^{32}$ This could provide new information about the natural history of secundum ASD which may be taken into account when assessing a patient for percutaneous ASD occlusion using a device placement.

The relation between measurements by three dimensional echocardiography and magnetic resonance imaging or surgery was good and within acceptable limits for clinical application. The maximum ASD dimension measured by three dimensional echocardiography correlated well with both magnetic resonance imaging and surgery. The systematic error for the group as a whole was slightly lower for Doppler imaging than for grey scale imaging when compared to both magnetic resonance imaging (25\% v 27\%, respectively) and surgery $(22 \% v 29 \%)$. This was not verified for the two age subgroups (table 2) because of the relatively small sample size. Correlation of the distances from the ASD rim to inferior vena cava, superior vena cava, tricuspid valve, and coronary sinus measured by three dimensional echocardiography and surgery in late ventricular diastole was also good, and again slightly favoured Doppler myocardial imaging over grey scale imaging.

\section{LIMITATIONS}

Technical limitations

Despite growing interest and extensive research in developing real time three dimensional echocardiography, current three dimensional reconstructions are available off-line only. In this particular study time was of minor importance as the information on ASD morphology was needed as a baseline to plan the repair strategy. The average acquisition time of a single dataset is approximately two to three minutes. The time required for the off-line processing of the ultrasound data may take up to 20 minutes. Finally, the time required to reconstruct the data in three dimensions differs according to the quality of the acquired ultrasound images and may take from two minutes to 20 minutes, and sometimes in a complicated case even longer.

Using this three dimensional system, the position of the ultrasound transducer during the data acquisition is calculated according to the mechanical steering logic and not according to the transducer spatial coordinates. Therefore the three dimensional system does not record unexpected changes in the transducer position which may create a rotational artefact. In such cases any attempt at taking measurements from the reconstructed three dimensional image should be abandoned. In this study to avoid problems related to the rotational artefact, each dataset was acquired twice, thus extending the acquisition time.

Care also needs to be taken during the adjustment of image gain settings. The three dimensional system is sensitive enough to reconstruct the ultrasound noise if such is left on the image. This will result in insufficiently clear three dimensional reconstruction.

\section{Methodological limitations}

In this study the information on ASD sizing was compared to that obtained from magnetic resonance imaging or surgery. Taking measurement from magnetic resonance imaging phase contrast images, one has to make sure that the shunt flow is orthogonal to the cine imaging plane. This is because the technique depends on flow related enhancement and phase contrast effects. In most cases the shunt orifice is best seen at end systole or early diastole only. It is difficult to assess the dynamic change in ASD size during the cardiac cycle reliably. Therefore, in this study only the maximum ASD dimensions (horizontal and vertical) were measured from magnetic resonance images. One might also expect the defect size to be overestimated if measured from images acquired upstream of the orifice where the jet converges, as well as downstream where it diverges.

Surgical closure of an ASD is usually performed using a cardioplegic solution achieving diastolic arrest of the heart. This unfortunately does not reflect the in vivo situation of the beating heart. The relaxed state of the heart tends to overestimate the size of the defect and the various distances measured. In this study, we elected to perform the surgical closure in a fibrillating heart, thus maintaining the cardiac tone. The measurements taken at surgery were therefore a more accurate reflection of the in vivo situation. In order to avoid interobserver variability, all measurements at surgery were undertaken by a single surgeon. Two independent measurements were obtained initially to avoid interobserver variability. However, it soon became apparent that there was little difference in the two observations.

\section{CONCLUSIONS}

Transthoracic three dimensional grey scale images and Doppler myocardial imaging both accurately displayed the varying morphology, 
dimensions, and spatial relations of ASDs. For the group as a whole there was no difference between the two ultrasound techniques in the accuracy of the reconstructed three dimensional images. However, in adult ASD patients, Doppler myocardial imaging was more effective than grey scale imaging in reconstructing a surgical view of ASDs. This study shows that a dynamic en face three dimensional image of an ASD is no longer restricted to the one seen only by a surgeon during an ASD repair, but may be reconstructed through the closed chest before closure of the defect. This should help to plan the surgical strategy or, where applicable, facilitate the selection of patients for percutaneous device closure.

This study was supported by the Chest, Heart and Stroke Association (196RR33163).

1 Dickinson DF, Arnold R, Wilkinson JL. Congenital heart disease among 160480 liveborn children in Liverpool 1960
to 1969 . Implications for surgical treatment. Br Heart 7 to 1969 . Implicati

2 Ward C. Secundum atrial septal defect: routine surgical treatment is not of proven benefit. Br Heart $\mathcal{F} 1994 ; 71: 219$ 23.

3 Burke RP, Michielon G, Wernovsky G. Video-assisted cardioscopy in congenital heart operations. Ann Thorac Surg 1994;58:864-8.

4 Schwartz DS, Ribakove GH, Grossi EA, Stevens JH, Siegel LC, St Goar FG, et al. Minimally invasive cardiopulmonary bypass with cardioplegic arrest: a closed chest technique with equivalent myocardial protection. 7 Thorac Cardiovas with equivalent myocar.

5 Rome JJ, Keane JF, Perry SB, Spevak PJ, Lock JE. Doubleumbrella closure of atrial defects. Initial clinical applications. Circulation 1990;82:751-8.

6 Das GS, Voss G, Jarvis G, Wyche K, Gunther R, Wilson RF. Experimental atrial septal defect closure with a new, Experimental atrial septal defect closure with a new,
transcatheter, self-centering device. Circulation 1993;88: transcath

7 Boutin C, Musewe NN, Smallhorn JF, Dyck JD, Kobayashi T, Benson LN. Echocardiographic follow-up of atrial septal defect after catheter closure by double-umbrella device. Circulation 1993;88:621-7.

8 Lloyd TR, Rao S, Beekman RH, Mendelsohn AM, Sideris EB. Atrial septal defect occlusion with the buttoned device (a multinstitutional US trial). Am f Cardiol 1994;73:28691.

9 Chan KC, Godman MJ. Morphological variations of fossa ovalis atrial septal defects (secundum): feasibility for transcutaneous closure with the clam-shell device. Br Heart $f$ 1993;69:52-5.

10 Magni G, Hijazi ZM, Marx G, Das G, Delabays A, Levine J, et al. Utility of 3-D echocardiography in patient selection and guidance for atrial saral defect (ASD) closure by the and guidance for atrial new Das-angel wings occluder

11 Schapira JN, Martin RP, Fowles RE, Popp RL. Single and two-dimensional echocardiographic features of the intera-
trial septum in normal subjects and patients with an atrial trial septum in normal subjects and patients
septal defect. Am f Cardiol 1979;43:816-19.

12 Morimoto K, Matsuzaki M, Tohma Y, Shiro O, Tanaka N, Michishige $\mathrm{H}$, et al. Diagnosis and quantitative evaluation of secundum-type atrial septal defect by transoesophageal Doppler echocardiography. Am f Cardiol 1990;66:85-91.

13 Hellenbrand WE, Fahey JT, McGowan FX, Weltin GG, Kleinman CS. Transesophageal echocardiographic guidance of transcatheter closure of atrial septal defect. $A m \mathcal{F}$ Cardiol 1990;66:207-13.
14 Mehta RH, Helmcke F, Nanda NC, Pinheiro L, Samdarshi TE, Vinod KS. Uses and limitations of transthoracic echocardiography in the assessment of atrial septal defect in the adult. Am F Cardiol 1991;67:288-94.

15 Holmvang G, Palacios IF, Vlahakes GJ, Dinsmore RE, Miller SW, Liberthson RR, et al. Imaging and sizing of atrial septal defects by magnetic resonance. Circulation 1995;92:3473-80.

16 Diethelm L, Dery R, Lipton MJ, Higgins CB. Atrial level shunts: sensitivity and specificity of MR diagnosis. Radiology 1987;162:181-6.

17 Reeder GS, Hagler DJ, Tajik AJ. Sensitivity of twodimensional echocardiography in direct visualization of atrial septal defect utilizing the subcostal approach: experience with 154 patients. F Am Coll Cardiol 1983;2: $127-35$.

18 King DL, Harrison MR, King DL, Gopal AS, Martin RP, DeMaria AN. Improved reproducibility of left atrial and left ventricular measurements by guided three-dimensional echocardiography. 7 Am Coll Cardiol 1992;20:1238-45.

19 Belohlavek M, Foley DA, Gerber TC, Greenleaf JF, Seward JB. Three-dimensional ultrasound imaging of the atrial septum: normal and pathologic anatomy. $\mathcal{F}$ Am Coll Cardiol 1993;22:1673-8.

20 Marx GR, Fulton DR, Pandian NG, Vogel M, Cao Q-L, Ludomirsky A, et al. Delineation of site, relative size and dynamic geometry of atrial septal defects by real-time 1995;25:482-90.

21 Lange A, Wright RA, Al-Nafusi A, Sang C, Palka P, Sutherland GR. Doppler myocardial imaging: a potential better method of data acquisition for three-dimensional echocardiography. F Am Soc Echocardiogr 1996;9:918-21.

22 Fleming AD, McDicken WN, Sutherland GR, Hoskins PR. Assessment of colour Doppler tissue imaging using test-phantoms. Ultrasound Med Biol 1994;20:937-57.

23 Azevedo J, Garcia-Fernandez M, Puerta P, Moreno M, SanRoman D, Torrecilla E, et al. Dynamic 3-Dimensional echocardiographic reconstruction of the left ventricle using color Doppler myocardial tissue imaging technique. In vivo experimental and clinical study [abstract]. F Am Coll Cardiol 1996;27(suppl A):901-47.

24 Lange A, Anderson T, Bouki KP, Fenn LN, Palka P, McDicken WN, et al. Validation of volume measurements by 3-dimensional echocardiography using Doppler myocardial imaging technique: in vitro and in vivo study [abstract]. Circulation 1995;92(suppl):I-798.

25 Altman DG, Bland JM. Measurement in medicine: the analysis of method comparison studies. Statistician 1983; 32:307-17.

26 Bland JM, Altman DG. Statistical methods for assessing agreement between two methods of clinical measurement. Lancet 1986;i:307-10.

27 Dinsomore RE, Wismer GL, Guyer D, Thompson R, Liu P, Stratemeier E, et al. Magnetic resonance imaging of the interatrial septum and atrial septal defects. Am f Roentgenol 1985;145:697-703.

28 Sakakibara M, Kobayashi S, Imai H, Watanabe S, Masuda Y, Inagaki Y. Diagnosis of atrial septal defect using Y, Inagaki Y. Diagnosis of atrial septal defect using

29 Sheikh KH, Smith SW, von Ramm OT, Kisslo J. Real-time, three-dimensional echocardiography: feasibility an initial use. Echocardiography 1991;8:119-25.

30 Pandian NG, Nanda NC, Schwartz SL, Fan P, Cao Q-L, Sanyal R, et al. Three-dimensional and four-dimensional transesophageal echocardiographic imaging of the heart and aorta in humans using a computed tomographic imaging probe. Echocardiography 1992;9:677-87.

31 Roelandt JRTC, ten Cate FJ, Vletter WB, Taams MA. Ultrasonic dynamic three-dimensional visualization of the heart with a multiplane transoesophageal imaging transducer . F Am Soc Echocardiogr 1994;7:217-29.

32 Franke A, Rulands D, Kuhl HP, Breithardt OA, Erena C, Grabitz RG, et al. Changes of the atrial septal defect area during the cardiac cycle: evaluation by dynamic transoesophageal three-dimensional echo [abstract]. Eur Heart f 1996;17(suppl):2317. 